\title{
Examining Purchase Intention of Eco-Friendly Products: A Comparative Study
}

\author{
Jiyoung Yoon, Soonhee Joung \\ Department of Consumer Studies, Ewha Womans University, Seoul, Korea \\ Corresponding author: jsh@ewha.ac.kr
}

\begin{abstract}
This is a comparative study to examine the process toward purchase intention of eco-friendly products with Korean and Chinese. This study extended the theory of planned behavior that explains men and women behavior also intention to performance the behavior. Furthermore, it established a conceptual framework in which environmental knowledge exerts an effect on attitude, perceived behavioral control and subjective norm, toward eco-friendly products, which in turn lead to the dependent variable, purchase intention of eco-friendly products. The results with 236 Korean and 315 Chinese college students showed that environmental knowledge positively affected eco-friendly subjective norm and attitude. Furthermore, subjective norm turned out to have the strongest influence on purchase intention of eco-friendly products in both the countries. On the other hand, although the two countries are similar oriental cultures, in the Korean case the path from perceived behavioral control to purchase intention was rejected but in the Chinese case a path from environmental knowledge to perceived behavioral control was so. That is, this research also found such differences in the paths toward purchase intention. These findings suggest the importance of environmental knowledge education to induce purchasing of ecofriendly products, and have practical implications, based on the differences between the two countries.
\end{abstract}

Keywords: Purchase intention, cross-culture, eco-friendly products, environmental knowledge, South Korea and China. 


\section{Introduction}

In the IPCC (Intergovernmental Panel on Climate Change), which was held in Korean in 2018, 195 countries approved the report that the increase in global warming will be limited to $1.5^{\circ} \mathrm{C}$ by 2100 (IPCC, 2018). This was to inform the world that the Earth's environment faced serious situations and to emphasize human conscious behavior for the environment. It means that consumption taking the environment into account is no longer considered to be only ethically good but must be practiced for the survival of the future and present age.

Although recent studies showed that Korean consumers have taken more interest in eco-friendly consumption than in the past, the percentage that such interest actually leads to eco-friendly purchase intention and behavior is still low (Statistics Korea, 2016; Yoon, 2019). In contrast, recent studies conducted in Europe reported that more than $72 \%$ of the European participants were seriously worried about recent climate change and more than $61 \%$ of them actually had experience to purchase eco-friendly products (Hanson, 2013; Ko and Jin, 2015). The reason that in this way eco-friendly purchase behavior varies among countries may be due to the difference in the understanding of eco-friendly consumption among countries. That is, consumers' consciousness of eco-friendly products may differ among countries, according to cultural difference and economic levels. However, most studies on environmental issues paid attention to consumers of just one country rather than addressing differences between countries (Chen et al., 2006; Yoon, 2019).

Environmental issues cannot merely be considered problems of individual countries. Therefore, this study was conducted to compare purchase intention of eco-friendly products between Korean and Chinese consumers. The reason for selecting China for the comparison to Korea was that lately China has shown great concerns with environmental issues unlike in the past (Yoon and Joung, 2019).

Actually, even though China has been pointed out as one of the countries that have had the strongest effect on the global environment, in the past the Chinese government did not show great interest in and high responsibility for environmental problems (Yoon and Joung, 2019). However, recognizing the seriousness of environmental problems, the government brought the New Environmental Protection Law into effect in 2015, and has led radical changes in environmental attitudes and behavior of individuals and companies at the national level. Hence, it would be meaningful to conduct a comparative study between contemporary Chinese and Korean consumers. For this purpose, the study applied theory of planned behavior that enables comprehensive research of the explanatory variables of human intention and behavior, and presented a framework of 'an extended theory of planned behavior' which had environmental knowledge, which has been recognized to cause changes in environmental attitudes, as the antecedent variable. 
The purpose of this study was to conduct of the suggested framework of the process toward purchase intention of eco-friendly products, and to examine differences in the process between Korean and Chinese consumers (Yoon and Joung, 2019). The empirical results will contribute to helping us understand the academic and practical meaning of environmental knowledge. Further, the comparative analyses between Korean and Chinese consumers are expected to have practical implications, such as an empirical basis for legislative material for relevant environmental policies.

\section{Literature Review}

Theory of Planned Behavior. The theory of planned behavior postulates attitude, subjective norm, and perceived behavioral control as the antecedent variables that influence human behavior and intention to carry out the behavior (Ajzen, 1991). In addition, Ajzen (1991) pointed out that the power of explanation of the theory would be enhanced by modifying the paths in the theory and adding necessary variables. Further, Perugini and Bagozzi (2010) showed that it was possible to extend or deepen the theory, by adding suitable variables based on the validity of the theory. Accordingly, this study sought to comprehensively examine the process and mechanism toward purchase intention of eco-friendly products by including environmental knowledge, which has turned out to influence the existing main factors, as the antecedent variable.

Environmental Knowledge. This concept refers to "a general knowledge of facts, concepts, and relationships concerning the natural environment and its major ecosystems" (Fryxell and Lo, 2003, p. 45). Like this, environmental knowledge indicates the information for understanding and evaluating human activities that can affect the global environment (Laroche et al., 2002). Therefore, people with a highly environmental knowledge is highly tend to recognize the seriousness of environmental problems as well as concrete action plans to solve the problems, and actually elicit positive changes in environmental attitudes (Kaiser \& Urs Fuhrer, 2003). Thus, this included environmental knowledge as the antecedent variable of the process toward purchase intention of eco-friendly products and sought to investigate its effect.

\section{Conceptual Framework}

This research established an extended framework of the 'Theory of planned behavior' (as presented in [Figure 1]), and suggested environmental knowledge as the antecedent variable. Therefore, it suggested totally six hypotheses and investigated differences in the same paths of the framework between Korean and Chinese consumers.

The associations of environmental knowledge with attitude, subjective norm, and perceived behavioral control. Several prior studies (e.g., Kaiser et al., 1999; Chan, 
2001; Laroche et al., 2002) demonstrated that environmental knowledge had a significant and positive influence on environmental attitude. Environmental knowledge has been found to positively affect environmental attitude as well as eco-friendly behavioral process (Kaiser et al., 1999; Kim, 2015). Paul et al.(2016) asserted that a variety of environmental antecedent variables would contribute to an increase in attitude, subjective norm, and perceived behavioral control, and empirically showed that environmental knowledge was positively associated with subjective norm, which indicates an individual's motivation to meet important others' expectations. In addition, an increase in knowledge turned out to be associated with an increase in perceived behavioral control, which controls an individual's behavior selection (Barkhi et al., 2008). As such, various studies examined an extended model by adding environmental antecedent variables including environmental knowledge. Thus, this study suggested the following hypotheses concerning the associations of environmental knowledge with consumers' attitude, subjective norm, and perceived behavioral control toward ecofriendly products (Yoon and Joung, 2019):

H1: EK (environmental knowledge) will have a positive association with attitude toward EFP (eco-friendly products).

$\mathrm{H} 2$ : EK (environmental knowledge) will have a positive association with subjective norm toward EFP (eco-friendly products).

H3: EK (environmental knowledge) will have a positive association with perceived behavioral control toward EFP (eco-friendly products).

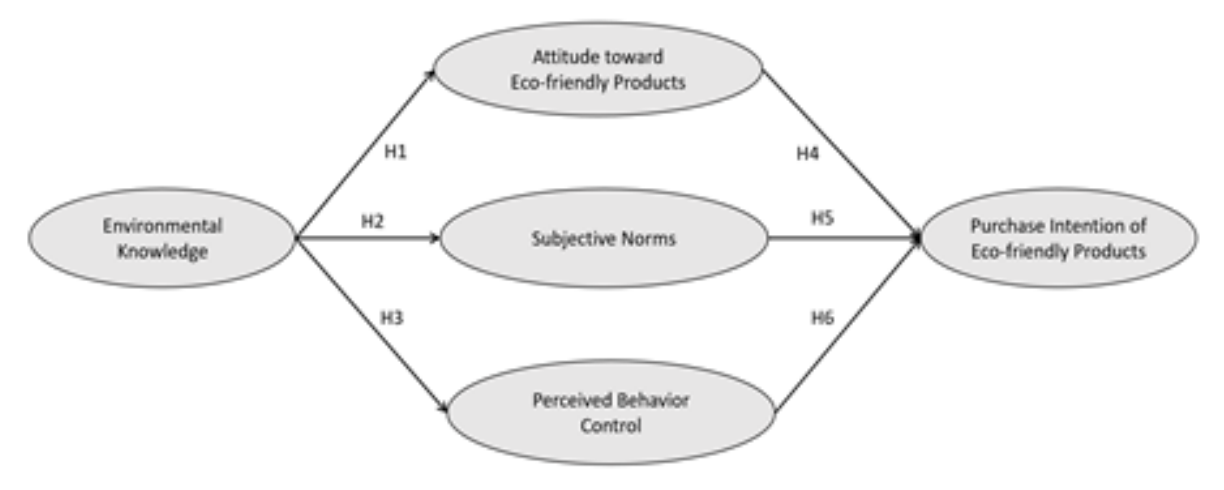

Fig. 1: The conceptual framework for this study.

The associations of attitude, subjective norm, and perceived behavioral control with purchase intention of eco-friendly products. In this research, the variables that are influenced by environmental knowledge are attitude, subjective norm, and perceived behavioral control, which were the antecedent variables in the theory of planned behavior. The relationship between attitude and purchase intention has turned out to be positive in various existing studies (Yoon and Joung, 2019). 
Perugini and Bagozzi (2001) demonstrated that subjective norm was an important positive determinant of behavior intention. Casalo et al. (2010) confirmed that attitude, subjective norm, and perceived behavioral control were the critical factors that predicted the strength of purchase intention in the positive direction. Furthermore, several studies of environmental behavior (e.g., Godin et al, 2008; Lam and Hsu, 2004) reported that human attitude, subjective norm, and perceived behavioral control were positive explanatory variables of environment-friendly purchase intention. Based on these previous studies, the following hypotheses were suggested:

H4: Attitude toward EFP (eco-friendly products) will have a positive association with purchase intention of the products.

H5: Subjective norm toward EFP (eco-friendly products) will have a positive association with purchase intention of the products.

H6: Perceived behavioral control toward EFP (eco-friendly products) will have a positive association with purchase intention of the products.

\section{Methodology}

Data collection. For the analyses, we collected the survey data from Korean and Chinese female college students in their twenties. The reason for selecting them as the research participants was that they have relatively more similar characteristics across countries, compared to the older age groups, in terms of socio-economic status (Peterson, 2001). Then, as the eco-friendly products for this study, we selected apparel, which is the shopping category that women in their twenties show the biggest interest in (Gardyn, 2002). Eco-friendly apparel products are operationally defined as apparel products using eco-friendly materials such as organic cotton rather than synthetic products and minimizing cloths and dyes in the production process, in the context of sustainable fashion. We explained this operationalized definition to the participants before starting the survey.

The survey was conducted in both the countries from April 10 to April 17, 2019. We administered an online survey with female college students living in both Seoul and Beijing. The online survey was conducted through email in Seoul and through an online survey site - that is, 问卷星—in Beijing. The same survey questions were used in both the cases. We collected totally 245 and 321 questionnaires and used 236 and 315 for the analyses in Korea and China, respectively. The excluded questionnaires included non-response, age younger or older than twenties, and the other gender - that is, male.

Study measures. We tried to improve the validity of the contents of the questionnaire items by drawing them from relevant previous studies and then supplementing and revising them based on the opinions of an expert group. In addition, we checked the reliability and validity of the measures through a pilot test (Chen, 2010; Yoon, 2019). Then, bilingual scholars were asked to review the final 
questionnaire and back-translate it into Korean, Chinese, and English (Yoon, 2019). We modified and used the following survey items for the study measures in order to make the items fit both the Korean and Chinese contexts: Ko and Jin's (2014) survey items for environmental knowledge, Armitage et al.'s (2001) for attitude and subjective norm toward eco-friendly products, and Paul et al.'s (2016) for perceived behavioral control toward and purchase intention of eco-friendly products. Finally, this study used 5 survey items for environmental knowledge, 5 for attitude, 3 for subjective norm, 4 for perceived behavioral control, and 3 for purchase intention. (All the survey items for each measure are presented in the Appendix.) All the survey items used a 7-point Likert scale. To test the reliability of each measure, Cronbach's $\alpha$ was calculated. Cronbach's $\alpha$ of each measure ranged from .802 to .938 , which showed that each measure had a satisfactory level of reliability.

\section{Empirical Analysis Results}

Using the SPSS 18.0 and AMOS statistical programs, the present study tested the hypotheses suggested above.

Data analysis. This analysis was conducted with three statistical procedures. First, CFA (confirmatory factor analysis) was conducted to check the reliability and validity of each model. Second, SEM (structural equation modeling) was carried out to test the suggested hypotheses. Third, a comparative analysis was conducted with the Korean and Chinese SEM results.

Data analysis results. Table 1 presents the reliability and validity test results of the measures in the measurement model. First, to check the appropriateness (or reliability) of the indicators (the survey items) for each variable, this study calculated SMC (squared multiple correlations) of all the indicators. Generally, it is recommended to exclude the indicators with an SMC less than .4; but as shown in Table 1, all the SMCs were greater than .4 , so all the indicators were considered to be appropriate overall. Second, we checked the convergent validity of all the variables with AVE (average variance extracted) and CR (construct reliability). The widely accepted standard has been 0.50 for AVE (Fornell and Larcker, 1981) and 0.70 for CR (Anderson and Gerbing, 1988). Table 1 also shows that the AVE and the CR of each latent variable were higher than the standards. Thus, the convergent validity of all the variables was verified.

Next, in order to test the suggested hypotheses, this study conducted a structural equation modelling (SEM) for each country case. The Korean model fitted well the data with relatively high fit indices: $\chi^{2}=978.76$, $\mathrm{df}=423, \mathrm{p}<.001$; GFI (Goodness of fit index $)=.912$; AGFI (adjusted goodness of fit index $)=.902$; NFI (Normed fit index $)=.952$; TLI $($ Tucker-Lewis index $)=.915$; CFI $($ comparative fit index $)=.941$; RMR (root mean squared residual) $=.059$; RMSEA (root mean squared error of approximation) $=.081$. Of the six hypotheses, except for the one concerning the path of PBC $\rightarrow$ PI, the five were statistically corroborated. 
Table 1: The results

\begin{tabular}{|c|c|c|c|c|c|c|c|}
\hline \multirow{2}{*}{$\begin{array}{c}\text { Latent } \\
\text { variable }\end{array}$} & \multirow{2}{*}{ Indicator } & \multicolumn{3}{|c|}{ South Korea } & \multicolumn{3}{|c|}{ China } \\
\hline & & SMC & CR & AVE & SMC & CR & AVE \\
\hline \multirow{5}{*}{ EK } & EK1 & .79 & \multirow{5}{*}{.87} & \multirow{5}{*}{.66} & .82 & \multirow{5}{*}{.82} & \multirow{5}{*}{.57} \\
\hline & EK2 & .75 & & & .84 & & \\
\hline & EK3 & .48 & & & .57 & & \\
\hline & EK4 & .69 & & & .70 & & \\
\hline & EK5 & .67 & & & .62 & & \\
\hline \multirow{5}{*}{ AT } & AT1 & .52 & \multirow{5}{*}{.87} & \multirow{5}{*}{.65} & .61 & \multirow{5}{*}{.87} & \multirow{5}{*}{.65} \\
\hline & AT2 & .58 & & & .45 & & \\
\hline & AT3 & .56 & & & .49 & & \\
\hline & AT4 & .68 & & & .52 & & \\
\hline & AT5 & .54 & & & .72 & & \\
\hline \multirow{3}{*}{$\mathrm{SN}$} & SN1 & .62 & \multirow{3}{*}{.80} & \multirow{3}{*}{.64} & .75 & \multirow{3}{*}{.80} & \multirow{3}{*}{.64} \\
\hline & $\mathrm{SN} 2$ & .45 & & & .43 & & \\
\hline & SN3 & .76 & & & .61 & & \\
\hline \multirow{4}{*}{ PBC } & $\mathrm{PBC} 1$ & .86 & \multirow{4}{*}{.76} & \multirow{4}{*}{.54} & .73 & \multirow{4}{*}{.62} & \multirow{4}{*}{.54} \\
\hline & $\mathrm{PBC} 2$ & .75 & & & .50 & & \\
\hline & PBC3 & .57 & & & .45 & & \\
\hline & $\mathrm{PBC} 4$ & .61 & & & .41 & & \\
\hline \multirow{3}{*}{ PI } & PI1 & .94 & \multirow{3}{*}{.92} & \multirow{3}{*}{.85} & .81 & \multirow{3}{*}{.87} & \multirow{3}{*}{.69} \\
\hline & PI2 & .91 & & & .77 & & \\
\hline & PI3 & .72 & & & .84 & & \\
\hline
\end{tabular}

Notes: SMC (squared multiple correlations); CR (construct reliability); AVE(average variance extracted); EK, (environmental knowledge); AT(attitude); SN(subjective norms); PBC(perceived behavioral control); PI( purchase intention) 
The Chinese model also fitted well the data with fit indices higher than the widely accepted standards: $x^{2}=1043.02$, df $=362, \mathrm{p}<.001$; GFI $=.926$; AGFI $=.945 ; \mathrm{NFI}=.971 ; \mathrm{TLI}=.985 ; \mathrm{CFI}=.952 ; \mathrm{RMR}=.043 ; \mathrm{RMSEA}=.044$. Also, of the six hypotheses, only one hypothesis concerning the path of EK $\rightarrow$ PBC was rejected. This was the different result with the Korean case (in Table 2 and Figure 2).

Table 2: The SEM results for testing H1- H6

\begin{tabular}{|c|c|c|c|c|c|c|c|c|c|}
\hline \multirow[b]{2}{*}{$\mathbf{H}$} & & & & \multicolumn{3}{|c|}{ South Korea } & \multicolumn{3}{|c|}{ China } \\
\hline & \multicolumn{3}{|c|}{ Path } & $\begin{array}{c}\text { Coeffic } \\
\text { ient }\end{array}$ & & Result & Coefficien & & \\
\hline H1 & \multirow{3}{*}{ EK } & $\rightarrow$ & AT & $\begin{array}{c}0.39 \\
(3.91)^{*} \\
*\end{array}$ & 0.12 & $\begin{array}{c}\text { Suppor } \\
\text { ted }\end{array}$ & $\begin{array}{c}0.25 \\
(3.39)^{* *}\end{array}$ & 0.06 & $\begin{array}{c}\text { Supporte } \\
\text { d }\end{array}$ \\
\hline $\mathrm{H} 2$ & & $\rightarrow$ & SN & $\begin{array}{c}0.26 \\
(3.58)^{*} \\
*\end{array}$ & 0.07 & $\begin{array}{c}\text { Suppor } \\
\text { ted }\end{array}$ & $\begin{array}{c}0.43 \\
(4.45)^{* *}\end{array}$ & 0.19 & $\begin{array}{c}\text { Supporte } \\
\text { d }\end{array}$ \\
\hline H3 & & $\rightarrow$ & $\begin{array}{c}\mathrm{PB} \\
\mathrm{C}\end{array}$ & $\begin{array}{c}0.53 \\
(8.12)^{*} \\
*\end{array}$ & 0.28 & $\begin{array}{c}\text { Suppor } \\
\text { ted }\end{array}$ & 0.17 (1.90) & 0.04 & Rejected \\
\hline $\mathrm{H} 4$ & $\mathrm{AT}$ & $\rightarrow$ & \multirow{3}{*}{ PI } & $\begin{array}{c}0.19 \\
(4.11)^{*} \\
*\end{array}$ & 0.04 & $\begin{array}{c}\text { Suppor } \\
\text { ted }\end{array}$ & $\begin{array}{c}0.28 \\
(3.42)^{* *}\end{array}$ & 0.08 & $\begin{array}{c}\text { Supporte } \\
\text { d }\end{array}$ \\
\hline H5 & SN & $\rightarrow$ & & $\begin{array}{c}0.57 \\
(8.78)^{*} \\
*\end{array}$ & 0.30 & $\begin{array}{c}\text { Suppor } \\
\text { ted }\end{array}$ & $\begin{array}{c}0.49 \\
(4.81)^{* *}\end{array}$ & 0.24 & $\begin{array}{c}\text { Supporte } \\
\text { d }\end{array}$ \\
\hline H6 & $\begin{array}{c}\mathrm{PB} \\
\mathrm{C}\end{array}$ & $\rightarrow$ & & $\begin{array}{c}-0.05 \\
(-1.24)\end{array}$ & 0.00 & $\begin{array}{c}\text { Rejecte } \\
\mathrm{d}\end{array}$ & $\begin{array}{c}0.31 \\
(3.30)^{* *}\end{array}$ & 0.10 & $\begin{array}{c}\text { Supporte } \\
\text { d }\end{array}$ \\
\hline
\end{tabular}

Notes: $* * \mathrm{p}<0.05$; EK (environmental knowledge); AT (attitude); SN (subjective norms); PBC (perceived behavioral control); PI (purchase intention). 


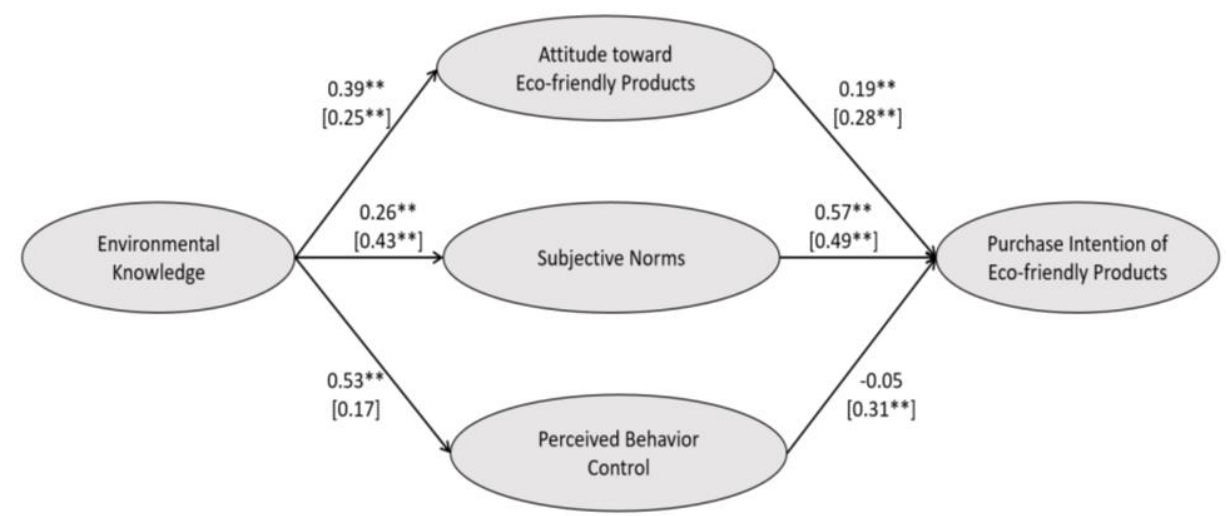

(Notes: $* * \mathrm{p}<.05$; the coefficients for the Chinese case are inside the parentheses.)

Fig. 2: The SEM results for the South Korean and Chinese cases.

\section{Discussions}

This study compared the process toward purchase intention of eco-friendly apparel products between the two countries: South Korea and China. For this purpose, it suggested a framework of an 'Extended theory of planned behavior' in which environmental knowledge was the antecedent variable, and investigated differences in the full paths towards purchase intention of the products with Korean and Chinese female college students.

The empirical results showed that environmental knowledge exerted a positive influence on attitude and subjective norm toward eco-friendly products for both the Korean and Chinese research participants. This suggests that an increase in environmental knowledge was likely to lead to more positive changes in ecofriendly attitude and subjective norm, which is a social factor. In addition, it was found that better eco-friendly attitude and subjective norm were likely to enhance purchase intention of eco-friendly products. Therefore, in both the countries, environmental knowledge education is expected to be highly effective for eliciting eco-friendly intention and behavior (Yoon and Joung, 2019).

On the other hand, a few differences were found between the Korean and Chinese cases. As presented above, this study suggested six hypotheses. However, the Korean data did not support solely H6 concerning the positive relationship between perceived behavioral control and purchase intention; but in the Chinese case, only H3 expecting the positive effect of environmental knowledge on perceived behavioral control was rejected.

In the Korean case, it was identified that abilities, access opportunities, availability, and economic power, which are suggested by or included in perceived behavioral control and which are considered to be controlled by individuals, did not increase purchase intention of eco-friendly products. This result is connected to recent studies on purchase intention of eco-friendly products conducted in Korea, 
and can be explained with the reasons that the interest in eco-friendly products is increasing but actual purchasing experience does not catch up with the interest. On the other hand, in the Chinese case, it was identified that while environmental knowledge had a positively meaningful effect on eco-friendly attitude and subjective norm, it did not so on perceived behavioral control, which is related to individuals' self-efficacy. Additionally, the effects of eco-friendly attitude, subjective norm, and perceived behavioral control on purchase intention were all found to be positive. Therefore, when they focus on attitudes and social issues in the educational contents about environmental knowledge, they can elicit more meaningful improvements. Next, looking into the effect sizes of the variables on purchase intention of eco-friendly products, in both the countries, subjective norm exerted the strongest effect, attitude was the next, and perceived behavioral control had the weakest influence.

The statistical results revealed that in both the countries subjective norm had the strongest influence on purchase intention of eco-friendly products. It can be inferred that this may result from the common point that both Korea and China are the culture areas in which people are highly affected by others close to themselves. Actually, in Western studies (e.g., Alwitt and Pitts, 1996; Ellen et al., 1991), attitude exerted a greater effect on behavior than subjective norm. This research showed a different result with these studies.

Thus, this study identified that both Korea and China are highly likely to elicit positive changes in eco-friendly purchasing process through environmental knowledge education. In particular, we can expect that if subjective norm leads to changes that call for environment-friendly actions across society, more people will practice eco-friendly purchasing. Furthermore, if people are inculcated with social responsibility, ethical citizenship, and solidarity consciousness, they are more likely to purchase eco-friendly products.

\section{Future Research}

This study is meaningful in that it compared Korean and Chinese consumers. However, because it chose a sampling method that restricted the age and gender of the research participants, the results had a limitation of generalization. Future research needs to be conducted with a wider range of ages and on a wider scale to overcome this limitation. In addition, both Korea and China are East Asian cultures with geographical and cultural similarities. In the future, it will be a developmental direction to carry out studies to add some countries of the Western culture. Further, besides the apparel products selected in this study, there are various eco-friendly products, and thus if more diverse products are chosen, it will be a more insightful study.

Despite of these limitations, this study is meaningful in that it made a comprehensive comparison of the process toward purchase intention of eco-friendly 
products between Korean and Chinese consumers. Theoretically, this study is expected to contribute to the future research related to the theory of planned behavior and environmental issues in that it extended theory of planned behavior by including environmental knowledge as the antecedent variable.

\section{References}

Alwitt, L. F., and Pitts, R. E. (1996). Predicting purchase intentions for an environmentally sensitive product. Journal of Consumer Psychology, 15(1), 49-64.

Anderson, J., and Gerbing, D. W. 1988. Structural Equation Modeling in Practice: A Review and Recommended Two-Step Approach. Psychological Bulletin, 103(3): 411-423.

Armitage, C. J., and Conner, M. (2001). Efficacy of the theory of planned behaviour: a meta-analytic review. British Journal of Social Psychology, 40, 471-499.

Ajzen, I. (1991). The theory of planned behavior organizational behavior and human decision Processes, 50, 179-211.

Barkhi, R., Belanger, F., and Hicks, J. (2008). A model of the determinants of purchasing from virtual stores. Journal of Organizational Computing and Electronic Commerce, 18(3), 177-196.

Chen, Y. S., Lai, S. B., and Wen, C. T. (2006). The influence of green innovation performance on corporate advantage in Taiwan. Journal of Business Ethics, 67(4), 331-339.

Diamantopoulos, A., Schlegelmilch, B. B., Sinkovics, R. R., and Bohlen, G. M. (2003). Can socio-demographics still play a role in profiling green consumers? A review of the evidence and an empirical investigation. Journal of Business Research, 56 (6), 465-480.

Ellen, P. S., Wiener, J. L., and Cobb-Walgren, C. (1991). The role of perceived consumer effectiveness in motivating environmentally conscious behaviors. Journal of Public Policy \& Marketing, 10(2), 102-117.

Fornell, C., and Larcker, D. F. (1981). Evaluating Structural Equation Models with Unobservable Variables and Measurement Error. Journal of Marketing Research, 39-50. 
Fryxell, G. E., and Lo, C. W. H. (2003). The influence of environmental knowledge and values on managerial behaviors on behalf of the environment: an empirical examination of managers in China. Journal of Business Ethics, 46(1), 45-69.

Gardyn, R. (2002). Educated consumers. American Demographics, 24(10), 18-19.

Gaston, G., Ariane B - G., Martin, E., and Jeremy, G. (2008). Healthcare professionals' intentions and behaviours: A systematic review of studies based on social cognitive theories. Implementation Science, 3(36), 1-12.

Hanson, C. B. (2013). Environmental concern, attitude toward green corporate practices, and green consumer behavior in the United States and Canada. American Society of Business and Behavioral Sciences eJournal, 9(1), 62-70.

IPCC Special Report on Global Warming of $1.5^{\circ} \mathrm{C}$, in the context of strengthening the global response to the threat of climate change. (2018). Intergovernmental Panel on Climate Change.

Ko, S. B., and Jin, B. (2017). Predictors of purchase intention toward green apparel products: A cross-cultural investigation in the USA and China. Journal of Fashion Marketing and Management: An International Journal, 21(1), 70-87.

Kaiser, F. G., Wolfing, S., and Fuhrer, U. (1999). Environmental attitude and ecological behaviour. Journal of Environmental Psychology, 19(1), 1-19.

Kaiser, F. G., and Gutscher, H. (2003). The proposition of a general version of the theory of planned behavior: predicting ecological behaviour. Journal of Applied Social Psychology, 33(3), 586-603.

Laroche, M., Tomiuk, M., Bergeron, J., and Barbaro-Forleo, G. (2002). Cultural differences in environmental knowledge, attitudes, and behaviors of Canadian consumers. Canadian Journal of Administrative Sciences, 19(3), 267-283.

Lam, A., and Hsu. T. (2006). Predicting behavioral intention of choosing a travel destination. Tourism Management, 27(4), 589-599

Paul, J., Modi, A., and Patel, J. 2016. Predicting green product consumption using theory of planned behavior and reasoned action. Journal of Retailing and Consumer Services, 29, 123-134. 
Peterson, R. A. (2001). On the use of college students in social science research: insights from a second-order meta-analysis. Journal of Consumer Research, 28(3), $450-461$.

Perugini, M., and Bagozzi, R. P. (2001). The role of desires and anticipated emotions in goal - directed behaviours: Broadening and deepening the theory of planned behaviour. The British Psychology Society. 40,79-98.

Statistics Korea. (2016). http://kostat.go.kr/wnsearch/search.jsp.

Yoon, J. (2019). A study on an eco-friendly product purchase process using expanded theory of planned behavior, Ph. D. Dissertation. Ewha Womans University, South Korea, 1-136.

Yoon, J., and Joung, S. (2019). A Study of Purchase Intention of Eco-friendly Products: A Cross-Cultural Investigation between Korea and China. International Journal of Smart Business and Technology, 7(2), 19-24. 